\title{
Glomerulosclerosis is transmitted by bone marrow-derived mesangial cell progenitors
}

\author{
Flavia Cornacchia, ${ }^{1}$ Alessia Fornoni, ${ }^{1}$ Anna Rita Plati, ${ }^{1}$ Alton Thomas, ${ }^{1}$ Yingcai Wang, ${ }^{1}$ \\ Luca Inverardi, ${ }^{2}$ Liliane J. Striker, ${ }^{1}$ and Gary E. Striker ${ }^{1}$ \\ ${ }^{1}$ Vascular Biology Institute, and \\ ${ }^{2}$ Diabetes Research Institute, University of Miami School of Medicine, Miami, Florida, USA \\ Address correspondence to: Gary E. Striker, University of Miami School of Medicine, Rosenstiel Medical Science Building, \\ 1600 NW Tenth Avenue, Room 1044 (R-104), Miami, Florida 33136, USA. \\ Phone: (305) 243-2811; Fax: (305) 243-2810; E-mail: gstriker@miami.edu.
}

Received for publication April 4, 2001, and accepted in revised form October 8, 2001.

We found that ROP Os/+ (Os/+) mice had diffuse glomerulosclerosis and glomerular hypertrophy and that their mesangial cells (the vascular smooth muscle cells of the glomerulus) displayed an apparent sclerosing phenotype. Since mesangial cells are the major source of scar tissue in glomerulosclerosis, we postulated that the sclerosis phenotype was carried by mesangial cell progenitors and that this phenotype could be derived from the bone marrow (BM). Therefore, we transplanted BM from $\mathrm{Os} /+$ mice into congenic $\mathrm{ROP}+/+$ mice $(+/+$ mice $)$, which have normal glomeruli. We found that glomeruli of $+/+$ recipients of $\mathrm{Os} /+$ marrow contained the Os/+ genotype, were hypertrophied, and contained increased extracellular matrix. Clones of recipient glomerular mesangial cells with the donor genotype were found in all $+/+$ recipients that developed mesangial sclerosis and glomerular hypertrophy, whereas $+/+$ recipients of $+/+B M$ had normal glomeruli. Thus, the sclerotic $(\mathrm{Os} /+)$ or normal $(+/+)$ genotype and phenotype were present in, and transmitted by, BM-derived progenitors. These data show that glomerular mesangial cell progenitors are derived from the BM and can deliver a disease phenotype to normal glomeruli. Glomerular lesions may therefore be perpetuated or aggravated, rather than resolved, by newly arriving progenitor cells exhibiting a disease phenotype.

J. Clin. Invest. 108:1649-1656 (2001). DOI:10.1172/JCI200112916.

\section{Introduction}

Phenotypic modulation is a common feature of vascular smooth muscle cells isolated from blood vessels that have been affected by progressive sclerosing diseases (1, 2 ). This is true of restenosis of vascular grafts, atherosclerosis, and glomerulosclerosis (2-4). Mesangial cells, the smooth muscle component of the glomerular vasculature, play a key role in the development of scarring in many progressive glomerular diseases (4). We found that glomerular mesangial cells exhibited stable phenotypic changes in vitro after the onset of diabetes in nonobese diabetic (NOD) mice, a glomerulosclerosisprone mouse strain that develops diabetic nephropathy (5-7). Identical phenotypic changes were found in several isolates of mesangial cells from the same kidney and in isolates from several different mice. Thus, stable phenotypic changes appeared to be a consistent finding in mesangial cells isolated from diabetic NOD mice that were developing a glomerulosclerotic lesion. Mesangial cells from $\mathrm{db} / \mathrm{db}$ mice (a model of nephropathy in type II diabetes) also demonstrated stable phenotypic changes after the onset of diabetes (8). We show herein that isolated mesangial cells of ROP Os/+ mice (Os/+ mice), a nondiabetic model of glomerulosclerosis, also exhibited stable in vitro phenotypic changes compared with their $\mathrm{ROP}+/+(+/+)$ littermates that had glomeruli of normal size and morphology (9).
Our results show that stable phenotypic changes are a common feature of mesangial cells in mice with a permissive genetic background. Since C57 Os/+ mice had normal glomeruli, we concluded that the Os mutation resulted in a sclerotic lesion only in the presence of a susceptible genetic background, and was not an independent cause of glomerulosclerosis (10).

Comparison between mesangial cells from sclerosisprone $+/+$ mice with mesangial cells derived from sclerosis-resistant $\mathrm{C} 57$ mice revealed marked differences in their extracellular matrix profile (11). Thus, mesangial cells in vitro retain many characteristics of the in vivo phenotype.

Because the sclerosis in the glomeruli of Os/+ mice was both diffuse and bilateral, as well as being localized to the mesangial region, we postulated that the cells carrying the sclerosing phenotype could originate from a common mesangial cell progenitor. Therefore, the three aims of the current study were to determine if mesangial cell progenitors were derived from the bone marrow (BM), if the donor phenotype was transmitted by mesangial cell progenitors, and if transplantation of mesangial cell progenitors from an animal with glomerulosclerosis transmitted the lesion to normal recipient glomeruli.

We used the Os/+ and +/+ strain combination for four reasons. First, while they are congenic, the two genotypes can be reliably differentiated (12). Secondly, 
ROP mice with the Os/+ genotype have progressive glomerulosclerosis, whereas those with the $+/+$ genotype do not $(9,13)$. Third, since the mesangial cells from both strains can be isolated and studied in vitro, allowing for identification of their genotype and phenotype, we were able to determine whether their progenitors were of BM origin. Finally, we would be able to determine whether transmission of the donor genotype and the donor phenotype were linked, and whether this resulted in the transmission of the donor lesion.

We found that mesangial cell progenitors originated from the $\mathrm{BM}$, and that $\mathrm{BM}$ from a mouse with glomerulosclerosis transmitted both the sclerotic lesions and glomerular hypertrophy to normal glomeruli. Thus, repair of injured blood vessels by progenitors carrying a disease phenotype could result in further progression of the disease, rather than its resolution.

In addition, we found that glomerular endothelial cells and aortic smooth muscle cell progenitors were also derived from the BM.

\section{Methods}

Animals. Female ROP Os/+ (Os/+) and ROP +/+ (+/+) mice, 6-8 weeks old, were obtained from The Jackson Laboratory (Bar Harbor, Maine, USA).

$B M$ transplantation. BM was obtained from 6- to 8week-old donor mice sacrificed using guidelines approved by the University of Miami Animal Care and Use Office. +/+ mice were lethally irradiated (950 cGy) and given $5 \times 10^{7} \mathrm{BM}$ cells intravenously. They were then followed for 8 weeks (14). All mice were 14-16 weeks old at sacrifice. No changes in general health status were noted in recipient mice.

Experimental and control groups. Ten $+/+$ mice received BM pooled from Os/+ mice, a congenic strain (15). +/+ and Os/+ mice share the $\mathrm{H}-2^{\mathrm{b}}$ haplotype (15). Two groups served as controls. Five $+/+$ mice received pooled $\mathrm{BM}$ cells from $+/+$ mice following irradiation. Four $+/+$ mice were irradiated and did not receive BM, to determine whether the irradiation dose chosen was lethal. All four of these mice died within two weeks.

Skin grafts. +/+ and Os/+ mice are thought to have identical H-2 loci. Since minor histocompatibility differences may have developed between these two strains, we examined Os/+ skin grafts placed on +/+ mice. Skin from the tails of Os/+ donors was engrafted to the backs of three $+/+$ recipients (16). The grafts were examined daily for evidence of sloughing, edema, and hair growth. The engrafted tail skin and a cuff of recipient skin was removed at 8 weeks. Cross sections including the donor skin and a margin of recipient skin were fixed in 10\% paraformaldehyde and embedded in paraffin. Sections were stained with hematoxylin and eosin. Organ preparation. Recipient mice were sacrificed 8 weeks after transplantation. The left kidneys were perfused with saline, and the upper and lower poles were removed and incubated in $0.1 \%$ collagenase (17). The remaining kidney was cut into small fragments, and either prepared for frozen sectioning or fixed in $10 \%$ formalin, embedded in methacrylate, sectioned, and stained with hematoxylin and eosin and periodic acid-Schiff. The spleen was frozen at $-80^{\circ} \mathrm{C}$ for genomic analysis, and a fragment of the aortic arch of each mouse was removed for cell culture.

Morphometry. Sections from methacrylate-embedded cortical tissue $(4 \mu \mathrm{m})$ were stained by the periodic acid-Schiff method. Digital images of 50 consecutive, randomly chosen glomeruli from each mouse were recorded with an Olympus BH-2 microscope and a MicroImage A209 RGB color video camera. Total glomerular volume $\left(\mu \mathrm{m}^{3}\right)$ was obtained using the MetaMorph image analysis computer program (Universal Imaging Corporation, West Chester, Pennsylvania, USA) (18).

Immunofluorescence microscopy. Type IV collagen was evaluated as previously described (4). For the identification of macrophages, sections were stained with a rat IgG anti-CD68 antibody (Serotec Inc., Raleigh, North Carolina, USA), followed by biotin-conjugated goat anti-rat IgG (Serotec Inc.), and streptavidin-conjugated FITC (Zymed Laboratories Inc., South San Francisco, California, USA). Spleen sections were used as a positive control. The negative control consisted of adjacent sections exposed only to the second antibody.

Microdissection of glomeruli. Glomeruli were separated from tubules and arterioles as described (17).

Isolation and cloning of glomerular mesangial and endothelial cells. Microdissected glomeruli were placed into wells of fibronectin-coated tissue culture plates and cultured in DMEM/F12 medium containing 20\% FBS $(19,20)$. We have previously described the characteristics of the three glomerular cell types (19). Mesangial cells were characterized by a number of features, including their elongated morphology, their growth in a "hill and valley" pattern, the presence of end-to-end filaments (revealed by staining with rhodamine-phalloidin), and immunostaining. We performed positive immunostaining with anti- $\alpha$-smooth muscle actin (DAKO Corp., Carpinteria, California, USA) (confirmed by RT-PCR for $\alpha$-smooth muscle actin) and anti-smooth muscle myosin (Biomedical Technologies Inc., Stoughton, Massachusetts, USA). Negative immunostaining was done with both mouse anti-endothelial cell monoclonal antibody IgG1 (cata$\log$ no. MAB16985; Chemicon International Inc., Temecula, California, USA) and an FITC-labeled antibody that recognizes macrophages (anti-mouse CD11b; Accurate Chemical \& Scientific Corp., Westbury, New York, USA) $(14,15)$. As expected, the small number of resident macrophages in the outgrowths $(<1-3 \%)$ did not survive past the first few days in culture. Endothelial cells were characterized by their cobblestone morphology, by rhodamine-phalloidin staining that showed peripheral staining but no end-to-end filaments, negative RT-PCR for $\alpha$-smooth muscle actin, positive immunostaining with a mouse anti-endothelial cell monoclonal antibody, and negative staining for mouse macrophages $(14,15)$. 
Glomerular cell outgrowths were obtained from the glomeruli of all ten mice receiving Os/+ BM for genotyping. Individual clusters of outgrowing cells were isolated with cloning rings and trypsinized, followed by single cell cloning and propagation (5). Twelve clones of mesangial cells were obtained from three of seven mice that had the Os/+ genotype in the initial outgrowths. Mesangial cells from one mouse in which the genotype of the early outgrowth was $+/+$ were cloned. Since endothelial cells were identified in the initial outgrowths from several recipients of Os/+ BM, the outgrowths were cloned to determine their genotype. Three glomerular endothelial cell clones were obtained from one $+/+$ recipient of Os/+ marrow.

Isolation and cloning of aortic smooth muscle cells. The aortic arches of three $+/+$ recipients of Os/+ BM were cut into $1-\mathrm{mm}^{3}$ fragments that were incubated for 1 hour at $37^{\circ} \mathrm{C}$ in a $0.1 \%$ collagenase solution. Smooth muscle cells were isolated and identified using the same conditions as described for glomerular mesangial cells. Five clones were obtained from these three recipients of Os/+ marrow. One clone cell was examined from each of two mice that had an Os/+ mesangial cell genotype in both first-passage cells and in several clones. In addition, three clone cells were examined from a mouse in which the mesangial cell clonal genotype was $+/+$.

$D N A$ analysis. DNA was extracted from the spleens, glomeruli, and isolated cells (12). Clonal populations of glomerular mesangial cells, glomerular endothelial cells, and aortic smooth muscle cells were examined. One million cells were incubated in a proteinase $\mathrm{K}$ solution at $60^{\circ} \mathrm{C}$ for 2 hours and then centrifuged at $4^{\circ} \mathrm{C}$. Phenolchloroform (Sigma-Aldrich, St. Louis, Missouri, USA) was added, and the aqueous phase was precipitated in ethanol and sodium acetate. DNA was prepared from cells from several different passages, and from more than three samples of each cell line (from passages 3-15). We used PCR primers that amplify simple sequence repeats linked to the Os genotype on chromosome 8 (the mouse Chr 8 SSLP marker D8Mit104; MIT SSLP database ID no. MPC2609) to identify the genotype of DNA of mesangial cells and glomeruli from all ten recipients of Os/+ BM (12). PCR products were separated on a $2 \%$ agarose gel.

Matrix metalloproteinase- 2 and type IV collagen mRNA. Mesangial cells isolated from $+/+$ and Os/+ native mice were obtained and characterized as described (11). Those were compared with cells isolated from +/+ recipients of BM. Twenty-four hours prior to collection, medium was changed to DMEM/F12 supplemented with $0.1 \%$ FBS. mRNA was extracted from the cell layer with TRI Reagent (Molecular Research Center Inc., Cincinnati, Ohio, USA) as previously described (6). Reverse transcription was performed on $2 \mu \mathrm{g}$ of total RNA with a first-strand DNA synthesis kit (Roche Molecular Biochemicals, Indianapolis, Indiana, USA). Competitive PCR was performed for matrix metalloproteinase-2 (MMP-2) by adding decreasing amounts of mutant templates to glomerular cDNA, as previously described (17). Semiquantitative PCR was used to analyze type IV collagen mRNA, using GAPDH as a control (6). Experiments were performed in triplicate.

MMP-2 activity. Twenty-four hours prior to collection, medium was changed to DMEM/F12 supplemented with $0.1 \%$ FBS. Supernatants were collected 24 hours later, and cell number was determined. MMP-2 activity was assessed using $10 \%$ gelatin zymogram gels, as described previously (6). Briefly, the medium was diluted to normalize for cell number $(-25,000$ cells $/ \mathrm{ml})$ before the addition of $5 \times$ Laemmli buffer under nonreducing conditions. Following electrophoresis, gels were washed for 1 hour in $2.5 \%$ Triton X-100, and incubated for 18 hours in $50 \mathrm{mM}$ Tris buffer. The gels were stained with Coomassie blue and air dried. Densitometry using NIH Image 1.6 was used to analyze relative MMP-2 activity. Experiments were performed in triplicate. Considerable attention was paid to comparing cultures with cell layers that had similar cell density, since MMP-2 activity is density dependent (6).

Urine albumin/creatinine ratios. Urine was collected in siliconized tubes. For the albumin assay, urine was diluted 1:1,000 and measured in duplicate with the Mouse Albumin ELISA Quantitation Kit from Bethyl Laboratories Inc. (Montgomery, Texas, USA). Optical density values were obtained using the MRX microplate reader (Dynex Technologies Inc., Chantilly, Virginia, USA) at a wavelength of $450 \mathrm{~nm}$. Albumin values $(\mathrm{ng} / \mathrm{ml})$ were recorded as the mean of the duplicate values. Urine was diluted 1:10 and 1:20 to determine the creatinine concentration by optical density using the Stanbio Creatinine Kit (Stanbio Laboratory, San Antonio, Texas, USA). Creatinine ( $\mathrm{ng} / \mathrm{ml})$ values were recorded as the mean of duplicate values.

Statistical analysis. Data were expressed as mean \pm SD. For morphometric analysis, means were compared using an unpaired $t$ test with Welch's correction. For gene expression and MMP-2 activity analysis, variances were compared using ANOVA and the Tukey multiple comparison test.

\section{Results}

Transfer of BM from mice with glomerulosclerosis resulted in reconstitution of the bematopoietic system in all congenic and isogenic recipients. There was no mortality in $+/+$ recipients of either $+/+$ or Os/+ BM. We identified the Os and $+/+$ genotypes by PCR with the mouse Chr 8 SSLP marker D8Mit104 (12). ROP +/+ mice are homozygous for this locus (Figure 1a, lane 2), whereas Os/+ mice are heterozygous (Figure 1a, lane 3). DNA from the spleens of all +/+ recipients of Os/+ BM contained the Os genotype (Figure 1a, lanes 4 and 5), showing that donor BM engrafted all recipients. Further evidence of engraftment was the fact that all BM recipients survived, whereas all irradiated mice that were not transplanted died within 2 weeks.

$+/+$ mice were tolerant to Os/+donor skin grafts. For the purpose of confirming that there was tolerance between the two congenic strains, we placed skin grafts from the 
a

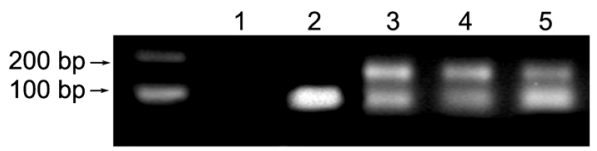

b

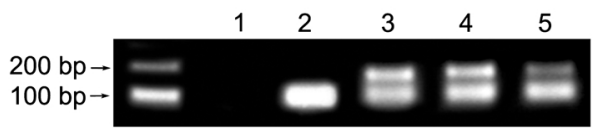

Figure 1

Genotype of spleen cells and isolated glomeruli. (a) PCR products of $+/+$ mice migrate as one band (lane 2), whereas those from Os/+ mice migrate as two bands (lane 3 ) in $2 \%$ agarose gels. DNA from the spleens of all $+/+$ recipients of Os/+ BM was found to contain the $\mathrm{Os} /+$ genotype (representative samples are shown in lanes 4 and 5). Lane 1 contains DNA not exposed to microsatellite primers, and the left lane represents bp size markers. (b) Shown are representative samples of DNA from glomeruli of nine of ten +/+ recipients of Os/+ BM (lanes 4 and 5). Lane 1 contains DNA not exposed to microsatellite primers, and the left lane represents bp size. Lanes 2 and 3 are similar to those in $\mathbf{a}$.

tails of Os/+ mice onto the backs of three $+/+$ mice and examined the grafts daily. There was no evidence of skin graft rejection by either serial visual examination or by review of histologic sections at the time of sacrifice 2 months after engraftment (data not shown).

Examination of recipient glomerular DNA revealed that the donor genotype was transmitted to nine of ten recipients. The $+/+$ genotype is recognized by a single band in a $2 \%$ agarose gel, whereas the Os/+ genotype is a doublet (Figure 1b, lanes 2 and 3). Glomeruli from all ten $+/+$ recipients of $\mathrm{Os} /+\mathrm{BM}$ were compared with those from naive $+/+$ and $\mathrm{Os} /+$ mice. The Os/+ genotype was identified in nine of ten $+/+$ recipients of Os/+ donor BM. The relative density of the two bands in agarose gels varied in recipients with the Os/+ genotype (Figure 1b, lanes 4 and 5). Mesangial cell isolates with the Os/+ genotype were more consistently obtained from mice in which the density of the Os band was more dense than the + band.

Transfer of BM from mice with glomerulosclerosis and glomerular bypertrophy resulted in development of glomerulosclerosis and glomerular bypertrophy in normal recipients. Os/+ mice with established glomerular lesions were used as BM donors (Figure 2a) $(9,10) .+/+$ mice were used as recipients because they are congenic with Os/+ mice (Figure 2c). +/+ mice that received Os/+ BM developed marked glomerular hypertrophy (2.5-fold increase in size, $P<0.001$ ) (Figure 3). Additionally, glomerulosclerosis, characterized by increased amounts of mesangial extracellular matrix, was comparable to that in the Os/+ donors. There was also marked enlargement of the mesangial spaces, which were shown by immunofluorescence microscopy to contain large accumulations of type IV collagen (Figure 4) $(9,10)$. The peripheral basement membranes appeared normal. The number of glomerular cells was not increased. The number of macrophages $(0.3 /$ glomerulus) was similar to that in $+/+$ recipients of $+/+$ marrow and native $+/+$ mice (data not shown). Thus, both glomerulosclerosis and glomerular hypertrophy were transmitted by BM-derived precursors.

Transfer of BM from mice with normal glomeruli to syngeneic recipients resulted in glomeruli of normal size and morphology. The glomeruli of $+/+$ recipients of $+/+$ BM were not different in morphology (Figure $2 \mathrm{~d}$ ) or size from $+/+$ control littermates (Figure 2c) $(9,10)$. Therefore, irradiation and transplantation were not the source of the glomerulosclerosis and/or glomerular hypertrophy found in $+/+$ recipients of Os/+ BM.

Mesangial cells of $+/+$ recipients of $O s /+B M$ expressed the $\mathrm{Os} /+$ genotype. We isolated individual glomeruli by microdissection to ensure that only resident glomerular cell types were represented in the outgrowing cells, i.e., mesangial, endothelial, or epithelial cells (podocytes) (19). Mesangial and endothelial cells, but not epithelial cells, were identified in the initial outgrowths. We identified the Os/+ genotype in the cells of seven of ten glomerular outgrowths from $+/+$ recipients of Os/+ BM (Figure 5). Twelve mesangial cell clones were studied from three of these outgrowths to determine their genotype. All had the Os/+ genotype. Hence, they were derived from the BM of the Os/+ donors. Three glomerular endothelial cell clones obtained from one of these mice revealed an Os/+ genotype, the same genotype present in the mesangial cells isolated from that mouse. Examination of DNA extracted from whole glomeruli revealed the Os/+ genotype in nine of ten

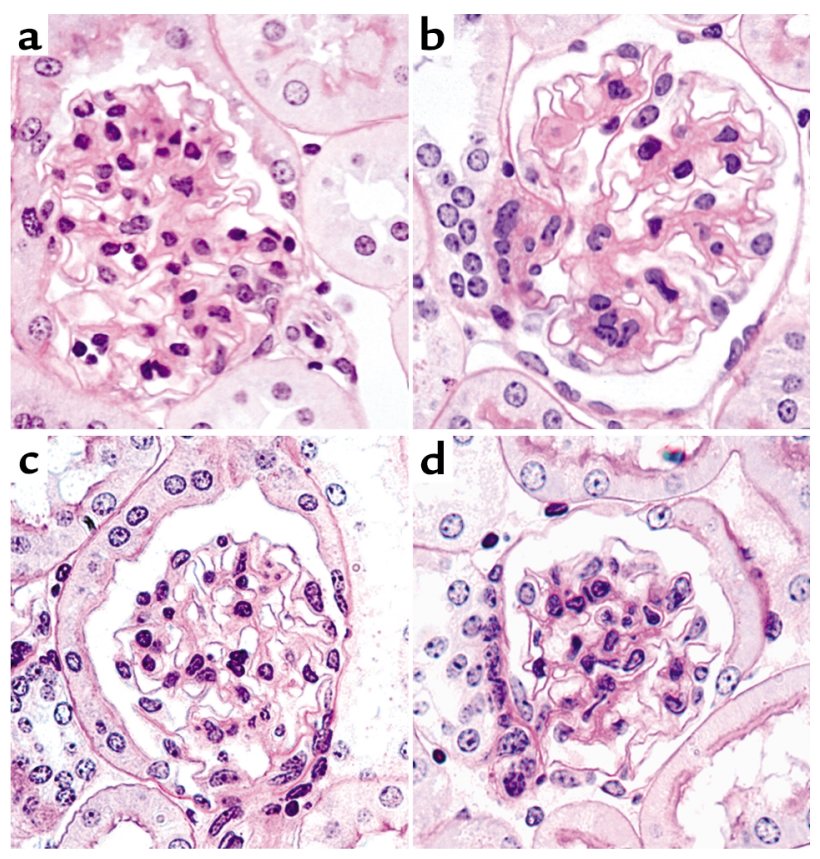

Figure 2

Glomerular histology. (a) Glomeruli of Os/+ donors are large and have a marked increase in mesangial matrix. (b) The glomeruli of $+/+$ recipients of Os/+ BM with the Os/+ genotype are large and have a marked increase in mesangial matrix. (c) Glomeruli of $+/+$ donors are normal in morphology and size. (d) Glomeruli are normal in $+/+$ recipients of +/+ BM. Periodic acid-Schiff stain. Original magnification: $\times 400$. 


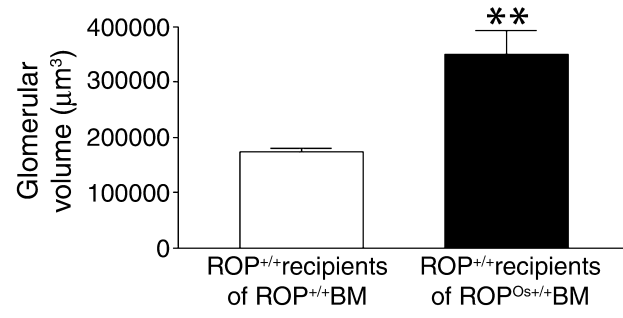

Figure 3

Morphometric analysis of glomerular volume. Glomerular volume of $+/+$ recipients of Os/+ BM is increased 2.5 -fold compared with +/+ recipients of $+/+\mathrm{BM}(P<0.001)$. ${ }^{*} P<0.001$.

mice. We were not able to isolate mesangial cells with the Os/+ genotype from two of these nine mice. We isolated five mesangial cell clones from the recipient lacking the Os/+ genotype in whole glomeruli to search for heterogeneity in the glomerular outgrowths, and found that all had the +/+ genotype.

Transfer of BM between congenic strains revealed that the donor mesangial cell genotype determined the mesangial cell phenotype in recipients. Mesangial cells from native kidneys of $+/+$ mice had a level of expression of MMP-2 mRNA that was twofold higher, as measured by competitive PCR (17) (Figure 6a) and enzyme activity (Figure $6, \mathrm{~b}$ and $\mathrm{c}$ ), than that of donor Os/+ mice. In contrast, the level of type IV collagen mRNA was identical in mesangial cells isolated from Os/+ and $+/+$ mice (data not shown), suggesting that an imbalance between synthesis and degradation could contribute to the development of glomerulosclerosis in Os/+ mice. The Os/+ MMP-2 phenotype was found in all of the recipient mesangial cell clones with the Os/+ genotype. In other words, the relative mRNA levels and levels of enzyme activity were not different between mesangial cells isolated from the kidneys of native Os/+ mice and those from +/+ recipients of Os/+ BM. Therefore, the Os/+ phenotype and genotype in mesangial cells correlated directly with the presence of glomerulosclerosis and glomerular hypertrophy in $+/+$ recipients of $\mathrm{Os} /+\mathrm{BM}$. The $+/+$ phenotype was found in mesangial cell clones isolated from the glomeruli of $+/+$ recipients of $+/+\mathrm{BM}$, all of which had normal glomeruli (data not shown).

Transfer of BM between congenic strains revealed that the genotype of aortic smooth muscle cells and mesangial cells may differ. Smooth muscle cells were isolated from the aortas of three +/+ recipients of Os/+ BM. Analysis of the genotype of first-passage cultures from the outgrowths of aortic fragments, and of all three clones from one of the primary cultures, revealed the Os/+ genotype (Figure 5). Thus, the aortic smooth muscle cells derived from explants were also derived from donor $\mathrm{BM}$-derived progenitors. The mesangial cells isolated from two of these three mice had the Os/+ genotype. Neither whole glomeruli nor mesangial cells isolated from the third mouse had the Os/+ genotype; in this mouse, the Os/+ genotype was found in the spleen and in aortic smooth muscle cells, but not in the glomeruli.
Urine albumin/creatinine ratios. We found no changes in the urine albumin excretion rate in mice sacrificed 8 weeks after $\mathrm{BM}$ transplantation (Os/+ vs. +/+ recipients: $0.041 \pm 0.007 \mathrm{mg} / \mathrm{g}$ vs. $0.030 \pm 0.009 \mathrm{mg} / \mathrm{g}$, no significant difference, respectively). We previously found that the albumin excretion rate was normal in 16-weekold Os/+ mice, but was increased by the age of 32 weeks (our unpublished data).

\section{Discussion}

Since ROP Os/+ mice, but not ROP +/+ mice, develop rapidly progressive glomerulosclerosis, this congenic strain combination was chosen for study of the repopulation of glomeruli by BM-derived progenitors $(9,10)$. The strains differ only at the Os locus on chromosome 8 , and the $+/+$ and Os/+ genotypes can be reliably differentiated $(12,15)$. Thus, we were able to determine whether the glomerular mesangial cell progenitors were derived from the BM and whether they transmitted the donor lesion following BM transplantation. Since additional mutations could have occurred in these well-established congenic strains, we examined skin from Os/+ mice grafted onto the $+/+$ mice, to ensure that the induction of glomerulosclerosis in $+/+$ recipients of Os/+ BM was not due to graft-versus-host disease. We found no evidence of skin graft rejection by gross or histologic examination. Hematopoietic reconstitution of recipients by donor BM was confirmed both by survival of all recipients and the finding that the spleen contained the donor genotype in all $+/+$ recipients of $\mathrm{Os} /+\mathrm{BM}$.
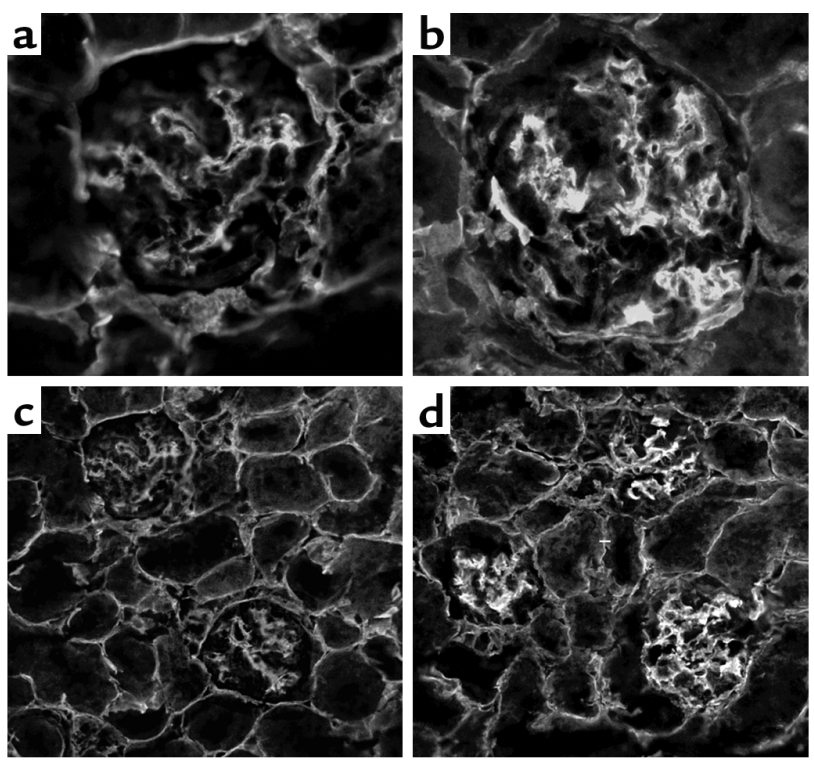

\section{Figure 4}

Immunofluorescence microscopy (type IV collagen). (a and c) $+/+$ recipients of $+/+B M$. The pattern of distribution is similar to that previously reported for native $+/+$ mice $(9,10)$. Original magnifications are $\times 400$ in $\mathbf{a}$ and $\times 400$ in $\mathbf{c}$. ( $\mathbf{b}$ and $\mathbf{d})+/+$ recipients of Os/+ BM. There are large aggregates of type IV collagen, similar in amount and distribution pattern to native Os/+ mice (9). Original magnifications are $\times 250$ in $\mathbf{b}$ and $\times 250$ in $\mathbf{d}$. 


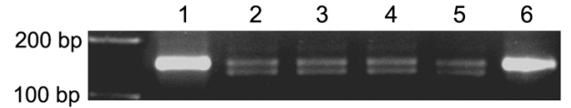

\section{Figure 5}

Genotype analysis of glomerular mesangial and endothelial cells and aortic smooth muscle cells. Representative samples: Lane 1:+/+ pattern. Lane 2: Os/+ pattern. Lane 3: The Os/+ donor genotype was found in mesangial cells isolated from seven of ten $+/+$ mice. Lane 4: The Os/+ genotype was found in all aortic smooth muscle cell clones. Lane 5: The Os/+ genotype was found in the glomerular endothelial cell clone. Lane 6: Mesangial cells had the recipient genotype $(+/+)$ in the single mouse in which the donor $(\mathrm{Os} /+)$ genotype was not detected in either whole glomeruli, in mesangial cell outgrowths, or in mesangial cell clones. However, the donor genotype was found in aortic smooth muscle cells and in the spleen in this mouse.

To ensure that only glomerular cells were studied in vitro, we isolated glomeruli by microdissection, and selected only those glomeruli that were free of capsules and arterioles (17). Thus, extracted DNA represented only glomerular constituents, and contamination of isolated cell preparations by nonglomerular sources was eliminated. The isolation of glomeruli by sieving techniques yields preparations containing $5-10 \%$ contamination by nonglomerular structures (4). Examination of DNA from glomeruli of all ten recipients revealed that nine of the ten contained the Os/+ genotype. Interestingly, the relative amounts of the $+/+$ and Os/+ genotype in $+/+$ recipients correlated with the ability to isolate mesangial cells with the Os/+ genotype. We found that the Os/+ genotype was invariably identified in mesangial cells from glomeruli that were the most sclerotic and enlarged. This correlated with a predominance of the Os genotype in agarose gels.

The Os/+ genotype was found in the glomeruli of nine of ten BM recipients, and in the initial cellular outgrowths from glomeruli of seven recipients. The Os/+ genotype was found in all twelve mesangial cell clones isolated from three of these seven mice. In two of the three recipients in which the Os/+ genotype was not identified in vitro, the relative density of the Os band in $2 \%$ agarose gels appeared to be decreased, compared with the relative density of bands from mice in which we were able to identify cells containing the Os/+ genotype in vitro. In the single mouse in which the phenotype of whole glomeruli was $+/+$, we found the $+/+$ genotype in both the initial cellular outgrowths from glomeruli and in five mesangial cell clones. Thus, it appeared that glomeruli in this mouse were not repopulated by donor progenitors. These data suggest that recipient glomeruli were not uniformly repopulated by donor progenitors.

We also studied clonal populations to determine whether the phenotype was concordant with the genotype, and whether the phenotype in recipients resembled that in the donors. We chose to study the MMP-2 profile, since others have reported that sclerosing glomerular diseases are associated with downregulation of MMP-2. For instance, MMP-2 was found to be decreased in glomeruli isolated from patients with dia- betic nephropathy (21). Furthermore, exposure of rat mesangial cells to elevated glucose levels resulted in a significant decrease in the levels of MMP-2 (22). We found that lower MMP-2 levels represented a stable phenotypic change in Os/+ mesangial cells that differentiated them from $+/+$ mesangial cells. Similarly, the levels of MMP-2 mRNA and activity were lower in mesangial cells isolated from Os/+ mice than in those from $+/+$ mice. We found that the phenotype of mesangial cells isolated from recipients correlated directly with the genotype, and did not differ from that found in the donor strains. Namely, mesangial cell MMP-2 mRNA and enzyme activity was lower in mesangial cells expressing the Os/+ genotype than in those that had the $+/+$ genotype. Furthermore, since there appeared to be no difference between the phenotype expressed in mesangial cells isolated from native and recipient kidneys, the events associated with BM transplantation did not appear to introduce artifacts into this aspect of the mesangial cell phenotype.

Furthermore, BM progenitors can deliver either a normal $(+/+)$ or a disease $(\mathrm{Os} /+)$ phenotype to

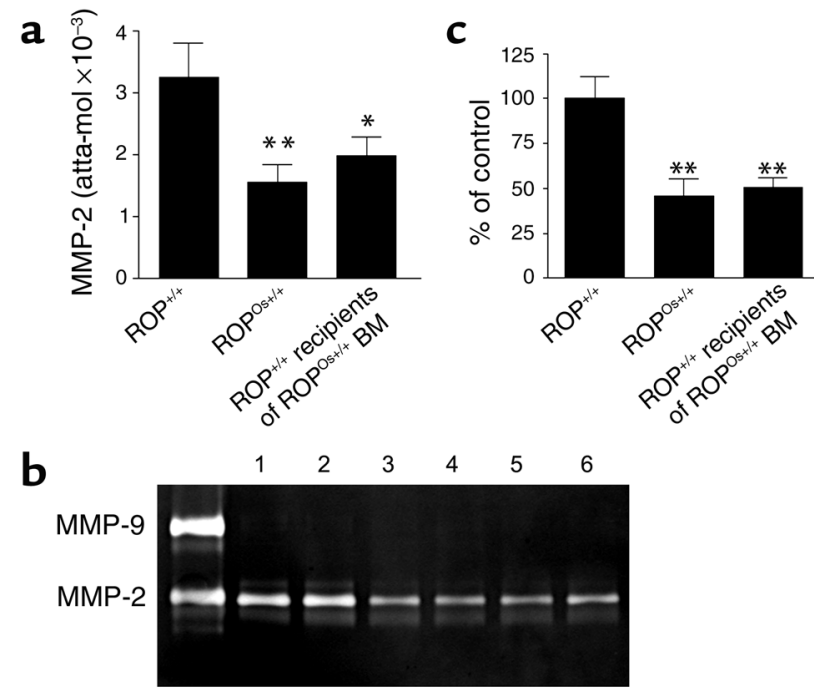

\section{Figure 6}

MMP-2 analysis. (a) MMP-2 mRNA expression in mesangial cells isolated from Os/+ mice and from $+/+$ recipients of Os/+ BM that had the Os/+ genotype was significantly lower than in mesangial cell clones from $+/+$ donor mice $\left({ }^{*} P<0.001\right.$ and $\left.{ }^{*} P<0.01\right)$. There were no differences between the levels of MMP- 2 mRNA in mesangial cell clones of donor Os/+ mice and levels in $+/+$ recipients of Os/+ BM that had the Os/+ genotype. (b) Zymography. Lanes 1 and 2: MMP-2 activity in mesangial cells from +/+ donors. Lanes 3 and 4: MMP-2 activity in mesangial cells isolated from $\mathrm{Os} /+$ donors. Lanes 5 and 6 : MMP- 2 activity in mesangial cell clones isolated from $+/+$ recipients of Os/+ BM (left lane contains molecular weight markers in the regions of MMP-2 and MMP-9). (c) MMP-2 activity in mesangial cell clones isolated from Os/+ donors and +/+ recipients of Os/+ BM was significantly lower than that in mesangial cell clones isolated from microdissected glomeruli of donor $+/+$ mice $\left({ }^{*} P<0.001\right)$. There were no differences in MMP-2 activity between mesangial cells from $\mathrm{Os} /+$ donors and mesangial cell clones with the Os/+ genotype that were isolated from $+/+$ recipients of Os/+ BM. 
glomeruli. Since mesangial cells play a key role in extracellular matrix accumulation, it is likely that the Os/+ mesangial cell progenitors were a major contributor to glomerular lesions in $+/+$ recipient mice. The absence of glomerular lesions in one $+/+$ recipient, and modest lesions in two others in which the degree of repopulation by Os/+ progenitors was diminished (as judged by genotype analysis of glomeruli) provides further evidence for this conclusion. However, we cannot rule out a role for a contribution by endothelial cells. We found no increase in the number of glomerular macrophages, suggesting that they were not a major factor in the development of glomerulosclerosis.

We expected to find both the $+/+$ and Os/+ genotypes in mesangial cells of Os/+ recipient BM. Since the $+/+$ genotype could have been obscured by the Os/+ genotype in mixed cultures of mesangial cells, we examined multiple clones of mesangial cells. We were unable to detect the $+/+$ genotype in mesangial cell clones from mice in which whole glomeruli and the initial glomerular outgrowths had the Os/+ genotype.

We could not isolate mesangial cells containing the Os/+ genotype from the glomeruli of three of ten $+/+$ recipients of $\mathrm{Os} /+\mathrm{BM}$, despite the fact that the Os/+ genotype was found in whole glomeruli in two of these three mice. Evidence that the BM transplantation was successful consisted of finding the Os/+ genotype in spleens of all recipients. The most likely explanation is that the number of progenitors repopulating glomeruli varied among mice. Those glomeruli with a preponderance of the Os/+ genotype had glomerular lesions, and we were able to isolate cells with this genotype from the cellular outgrowths. In contrast, the glomeruli of those in which the $+/+$ genotype predominated were affected to a lesser extent, and the isolated cells all had the $+/+$ genotype. Interestingly, in the three recipients of Os/+ marrow that had a $+/+$ mesangial cell genotype, we found only the Os/+ genotype in aortic explant outgrowths from all three mice and in three smooth muscle clones obtained from one of these aortic outgrowths.

There is increasing evidence that the BM is the source of progenitors for multiple organs. More than 20 years ago, we found that one of the fixed glomerular cells, the resident macrophage, was derived from the BM (23). Since the current work was submitted, others have also shown that mesangial cell progenitors can be derived from the BM in transgenic mice (24). Several laboratories have found that $\mathrm{BM}$ cells can differentiate into osteoblasts (25), adipocytes (26), and endothelial cells $(27,28)$. In addition, transplantation of marrow (stromal) cells has been successfully used in the treatment of patients with osteogenesis imperfecta, a genetic disorder of mesenchymal cells resulting from a mutation in one of the two genes encoding type I collagen (29). Recently, it has been shown that several types of mesenchymal cells, including interstitial fibroblasts, hepatocytes, osteoblasts, endothelial cells, and striated muscle cells, are derived from the BM, even in adult animals $(30-32)$. Marrow cells have been shown to differentiate into astrocytes after injection into neonatal mouse brain (33). Finally, a stem cell population common to both smooth muscle and endothelial cells has been identified (34). Thus, the BM is the site of progenitor cells for multiple tissues.

Mesangial cells isolated from microdissected glomeruli of mice with glomerulosclerosis have been shown to display a stable phenotypic change in vitro after diverse stimuli, including type 1 and type 2 diabetes, excess growth hormone, and cyclosporin administration (5-8, $11,20)$. Fibroblasts from the skin of patients with scleroderma or diabetes were also found to retain a pathologic phenotype, suggesting that phenotypic abnormalities persist both in vitro and in vivo, and affect both vascular and nonvascular mesenchymal cells $(35,36)$.

There has been controversy in the literature about whether BM-derived cells are capable of delivering both their genotype and phenotype to the mesenchymal tissues (37). We found that mesangial regions were reconstituted by BM-derived mesangial cell progenitors, and that the progenitors can carry both their genotype and phenotype to recipient glomeruli. Specifically, when the donor genotype was associated with glomerulosclerosis and glomerular hypertrophy, and donor progenitors localized to recipient glomeruli, the outcome was the induction of both glomerulosclerosis and glomerular hypertrophy. In contrast, when the donor genotype was associated with normal glomeruli, recipient glomeruli were normal in morphology and size.

Our data are the first to show that mesangial cell progenitors carrying a disease genotype and phenotype can be transmitted by BM-derived mesangial cell progenitors. These data may necessitate reconsideration of current pharmacological strategies in the management of chronic glomerulosclerosis, and possibly other chronic diseases of blood vessels.

\section{Acknowledgments}

This work was supported by NIH grants R01 AG17170-03 and R01 AG-17170-01S1 (to L.J. Striker), R03 AG-18618-01, R01 AG-19366-01, and R01 AG-1936601S1 (to G.E. Striker), and by Juvenile Diabetes Foundation International grant 1-2000-242 (to L. Inverardi). A.R. Plati is a recipient of a National Kidney Foundation of Florida Fellowship.

\footnotetext{
1. Owens, G.K. 1995. Regulation of differentiation of vascular smooth muscle cells. Physiol. Rev. 75:487-517.

2. Regan, C.P., Adam, P.J., Madsen, C.S., and Owens, G.K. 2000. Molecular mechanisms of decreased smooth muscle differentiation marker expression after vascular injury. J. Clin. Invest. 106:1139-1147.

3. Radke, P.W., et al. 1999. Mechanisms of acute lumen gain and recurrent restenosis after rotational atherectomy of diffuse in-stent restenosis: a quantitative angiographic and intravascular ultrasound study. J. Am. Coll. Cardiol. 34:33-39.

4. Striker, G.E., et al. 1995. Pathogenesis of nonimmune glomerulosclerosis: studies in animals and potential applications to humans. Lab. Invest. 73:596-605.

5. Elliot, S.J., et al. 1993. Mesangial cells from diabetic NOD mice constitutively secrete increased amounts of insulin-like growth factor-I. Endocrinology. 133:1783-1788.

6. Lupia, E., et al. 1999. IGF-1 decreases collagen degradation in diabetic NOD mesangial cells: implications for diabetic nephropathy. Diabetes. 48:1638-1644.
} 
7. Ardaillou, N., Placier, S., Striker, L., Striker, G., and Ardaillou, R. 1999. Mesangial cells from diabetic NOD mice constitutively express increased density of atrial natriuretic peptide $\mathrm{C}$ receptors. Kidney Int. 55:1293-1302

8. Oemar, B.S., Foellmer, H.G., Hodgdon-Anandant, L., and Rosenzweig, S.A. 1991. Regulation of insulin-like growth factor I receptors in diabetic mesangial cells. J. Biol. Chem. 266:2369-2373.

9. He, C.J., Zalups, R.K., Henderson, D.A., Striker, G.E., and Striker, L.J. 1995. Molecular analysis of spontaneous glomerulosclerosis in Os/+ mice, a model with reduced nephron mass. Am. J. Physiol. 269:F266-F273.

10. He, C.J., et al. 1996. Dissociation of glomerular hypertrophy, cell proliferation, and glomerulosclerosis in mouse strains heterozygous for a mutation (Os) which induces a $50 \%$ reduction in nephron number. J. Clin. Invest. 97:1242-1249.

11. Fornoni, A., et al. 2000. Matrix accumulation in mesangial cells exposed to cyclosporin A requires a permissive genetic background. Transplantation. 70:587-593.

12. Lenz, O., et al. 1998. Linkage disequilibrium mapping reveals suppressed recombination at the Os locus. Mamm. Genome. 9:681-682.

13.Zheng, F., Striker, G.E., Esposito, C., Lupia, E., and Striker, L.J. 1998 Strain differences rather than hyperglycemia determine the severity of glomerulosclerosis in mice. Kidney Int. 54:1999-2007.

14. Selvaggi, G., et al. 1995. Abrogation of graft-versus-host disease following allotransplantation of cytotoxically deficient bone marrow across major histocompatibility barriers. Ann. NY Acad. Sci. 770:339-344.

15. 2000. JAX GEMM strains: update on nomenclature changes for congenic mutant strains. JAX Bulletin. Number 4, May 2000 http://jaxmice.jax.org/html/nomenclature/nomen_memo.shtml.

16. Rosenberg, A.S. 1991. Skin allograft rejection. In Current protocols in immunology. J. Coligan, A. Kruisbeek, D. Margulies, E. Shevach, and W. Strober, editors. John Wiley \& Sons Inc. New York, New York, USA. 4.4.1.-4.4.12.

17. Peten, E.P., et al. 1992. Age-related changes in alpha 1- and alpha 2-chain type IV collagen mRNAs in adult mouse glomeruli: competitive PCR Am. J. Physiol. 263:F951-F957.

18. Pesce, C.M., Striker, L.J., Peten, E., Elliot, S.J., and Striker, G.E. 1991 Glomerulosclerosis at both early and late stages is associated with increased cell turnover in mice transgenic for growth hormone. Lab. Invest. 65:601-605.

19. Mackay, K., et al. 1988. Glomerular epithelial, mesangial, and endothelial cell lines from transgenic mice. Kidney Int. 33:677-684.

20. Jacot, T.A., Striker, G.E., Stetler-Stevenson, M., and Striker, L.J. 1996. Mesangial cells from transgenic mice with progressive glomerulosclerosis exhibit stable, phenotypic changes including undetectable MMP-9 and increased type IV collagen. Lab. Invest. 75:791-799.

21. Del Prete, D., et al. 1997. Down-regulation of glomerular matrix metalloproteinase-2 gene in human NIDDM. Diabetologia. 40:1449-1454.

22. Leehey, D.J., Song, R.H., Alavi, N., and Singh, A.K. 1995. Decreased degradative enzymes in mesangial cells cultured in high glucose media. Diabetes. 44:929-935.

23. Striker, G.E., Mannik, M., and Tung, M.Y. 1979. Role of marrow-derived monocytes and mesangial cells in removal of immune complexes from renal glomeruli. J. Exp. Med. 149:127-136.

24. Imasawa, T., et al. 2001. The potential of bone marrow-derived cells to differentiate to glomerular mesangial cells. J. Am. Soc. Nephrol. 12:1401-1409.

25. Rickard, D., et al. 1996. Isolation and characterization of osteoblast precursor cells from human bone marrow. J. Bone Miner. Res. 11:312-324

26. Bennett, J.H., Joyner, C.J., Triffitt, J.T., and Owen, M.E. 1991. Adipocytic cells cultured from marrow have osteogenic potential. J. Cell Sci. 99:131-139.

27. Asahara, T., et al. 1997. Isolation of putative progenitor endothelial cells for angiogenesis. Science. 275:964-967.

28. Schatteman, G.C., Hanlon, H.D., Jiao, C., Dodds, S.G., and Christy, B.A. 2000 . Blood derived angioblasts accelerate blood-flow restoration in diabetic mice. J. Clin. Invest. 106:571-578.

29. Horwitz, E.M., et al. 1999. Transplantability and therapeutic effects of bone marrow-derived mesenchymal cells in children with osteogenesis imperfecta. Nat. Med. 5:309-313.

30. Prockop, D.J. 1997. Marrow stromal cells as stem cells for nonhematopoietic tissues. Science. 276:71-74.

31. Petersen, B.E., et al. 1999. Bone marrow as a potential source of hepatic oval cells. Science. 284:1168-1170.

32. Ferrari, G., et al. 1998. Muscle regeneration by bone marrow-derived myogenic progenitors. Science. 279:1528-1530.

33. Kopen, G.C., Prockop, D.J., and Phinney, D.G. 1999. Marrow stromal cells migrate throughout forebrain and cerebellum, and they differentiate into astrocytes after injection into neonatal mouse brains. Proc. Natl. Acad. Sci. USA. 96:10711-10716.

34. Yamashita, J., et al. 2000. Flk-1-positive cells derived from embryonic stem cells serve as vascular progenitors. Nature. 408:92-96.

35. Strehlow, D., Jelaska, A., Strehlow, K., and Korn, J.H. 1999. A potential role for protease nexin 1 overexpression in the pathogenesis of scleroderma. J. Clin. Invest. 103:1179-1190.

36. Trevisan, R., et al. 1992. Na+/H+ antiport activity and cell growth in cultured skin fibroblasts of IDDM patients with nephropathy. Diabetes. 41:1239-1246.

37. Bianco, P., and Robey, P.G. 2000. Marrow stromal stem cells. J. Clin. Invest. 105:1663-1668 